\title{
HABITS AND REASONS OF DELAYED PRESENTATION OF PATIENTS WITH ORAL CANCER AT A TERTIARY CARE HOSPITAL OF A THIRD WORLD COUNTRY
}

\author{
Zohra Saleem 1 , Syed Akbar Abbas ${ }^{2}$, Falak Nadeem ${ }^{3}$, Muhammad Mansoor Majeed $^{4}$
}

\author{
${ }^{1}$ Postgraduate Trainee Department of Oral and Maxillofacial Surgery, Dow University of Health Sciences, Karachi, \\ Pakistan. \\ 2Otolaryngologist, Head \& Neck Surgeon, Patel Hospital, Karachi, Pakistan. \\ ${ }^{3}$ Dental Surgeon, Dow University of Health Sciences, Karachi, Pakistan. \\ ${ }^{4}$ Postgraduate Trainee Department of Oral Biology, Dow University of Health Sciences, Karachi, Pakistan. \\ Correspondence: Muhammad Mansoor Majeed, Cell: 0321-2661979 Email: mmansoormajeed@gmail.com
}

\begin{abstract}
Background: Oral cancer accounts for nearly one-fifth of all cancers in males and one-tenth in females globally. Squamous cell carcinoma accounts for $90 \%$ of the oral cancer. The occurrence rate of oral cancer is higher in Sri Lanka, Bangladesh, Pakistan and India. In South Asia oral cancer constitutes $25 \%$ of all new cancer reported among males. The estimated incidence of oral cancer in Pakistan is $14.7 \%$.

Methods: A cross sectional observational study was carried out at a tertiary care hospital in Karachi with a sample of 145 patients with pathological diagnosis of squamous cell carcinoma of oral cavity. Patients were characterized into early stage (I and II) and advanced stage (III and IV), according to AJCC classification of tumors (2010). Data entry and analysis was carried out on SPSS 19. Qualitative data was expressed in percentages and results were accessed by Chi squared test.

Results: The mean age was recorded $47.87 \pm 12.50$ years. Males participants were $63.4 \%$ and $36.6 \%$ females were incorporated in our study. More than half came with the Stage IV. 35.2\% of participants became aware of their initial symptom before 6 months before pursuing any doctor. 83\% patients presented with noteworthy habit of Tobacco or Chalia chewing. Bulk of the participants stated that they would have presented before if they had knowledge about the disease. $85 \%$ thought that increasing awareness of oral cancer through media may decrease the time in seeking help from qualified doctor.
\end{abstract}

Conclusion: The study concludes that most of the participants presented late to the professional because of lack of knowledge about signs and symptoms of oral malignancy.

Keywords: Squamous cell carcinoma, late presentation, oral cancer, oral habits

\section{Introduction}

Oral cancer accounts for nearly one-fifth of all cancers in males and one-tenth in females globally1. Squamous cell carcinoma accounts for $90 \%$ of the oral cancer 2 . The occurrence rate of oral cancer is higher in Sri Lanka, Bangladesh, Pakistan and India. In South Asia oral cancer constitutes $25 \%$ of all new cancer reported among males 3 . The estimated incidence of oral cancer in Pakistan is $14.7 \% .3$ Study reported that among all the reported cases of cancers ,21\% males and $11 \%$ females are reported with oral cancer in Pakistan 4. It is also suggested that oral cancer is the second and third most common cancer in females and males respectively in Pakistan5. According to the two year statistics of the Karachi Cancer Registry, SCC of the oral cavity is the second most common cancer in Karachi District South with an yearly incidence of $0.0041 \%$ in males and $0.0040 \%$ in females6. Use of pan, gutka, chalia and forms of tobacco in our population are the main cause of the disease burden.

Late presentation of cancer patient brings a great challenge to care providers which consequently results in advanced stage disease and subsequently high rate of recurrence and poor prognosis with high treatment cost7, 8. There are two reasons behind patient's deferral in consulting a health care professional, i.e., patient's delay in identifying the signs of the disease and hindrances in gaining access to professional care due to higher cost and a professional delay could be a cause of failure in identifying the signs and symptoms suggestive of cancer9, 10.The postponement in the cancer journey is defined as the patient's delay prior to seeing the right kind of healthcare professional9. Early detection has greater chances of better treatment outcomes and prognosis of disease. The key factor resulting in late presentation is lack of awareness about disease. Other 
factors that are associated with the delay are the financial conditions of the patient more over the attitude of patient towards his own health may have direct impact and relation with severity of the disease at the time of visiting health care professional and this may results in the prognosis of the disease $10-14$. Timing of diagnosis may also be influenced by the cultural beliefs and alternative medicine availability in our part of the world. Delayed diagnosis affects the treatment of the patients and produces an additional burden on the already miserable resources of patients and health care system.

This study will help to highlight the omissions in the patient's perception of the disease process and may serve to bring awareness among future patients and care providers in our part of the world.

\section{Methodology}

A cross sectional, observational study was carried out on patients with pathological diagnosis of squamous cell carcinoma of oral cavity. Study was conducted at a tertiary care teaching hospital in Karachi, Pakistan. The duration of this study was 6 months. A convenient sample of 145 patients presenting with oral cancer was obtained from Head and Neck Cancer data base maintained in the research office. This research was not financially supported by any source. The study proposal was approved by Patel hospital's ethics committee.

All patients with pathological diagnosis of squamous cell carcinoma of oral cavity presenting to Head and Neck service were included in the study. Patients with other malignancies (i.e. Iymphoma, sarcoma, minor salivary gland malignancies) and patients unwilling to comply were excluded from the study. Questionnaire was pretested on 20 patients to ensure the validity of the instrument and to approximate the length of the interview. Informed consent (according to Helsinki Declaration) was sought and assurance of confidentiality about personal data provided. Patients were interviewed through questionnaire in a comfortable environment by a research officer. Interview lasted for 10 minutes. Questionnaire was translated into local language for administration to patients. The first section of the questionnaire deals with the demographic variables which included age, sex, education, occupation. The second section comprising of 15 questions includes information regarding duration of symptoms, time of presentation to the HCP, treatment history, knowledge of risk perception and perception regarding spreading awareness.

Socioeconomic data was also collected. Information pertaining to the tumor site and size was obtained from the hospital head and neck cancer data base. Tumors were staged according to The American Joint Committee on Cancer classification15. Patients were categorized according to early stage (stage I \& stage II) and advanced stage (stage III \& stage IV). Data entry and analysis was carried out on SPSS 17 on computer based in research office. Qualitative data was summarized by using percentages and frequencies.
Non-Parametric T Test and Chi squared tests were used to evaluate significance level. Values were considered significant when they were below $0.05(p<0.05)$. Data was password protected and access limited to investigators and analyst only.

\section{Results}

Total 145 patients were incorporated after meeting the inclusion criteria were identified by participating health care professionals. Male patients were 93(64.1\%) and $52(35.8 \%)$ patients were female. The mean age of the participants was $44 \pm 5.8$ years. As per our study findings Age is not a significant factor in terms of developing cancer $(P=0.411)$. It was found that $66(45.5 \%)$ were employed or working on daily wages. In our study 97 $(66.9 \%)$ of the cancer patients were earning below 10000 RS a month or unemployed. $35(24.1 \%)$ were earning in the range of 10001 to 25000 PKR. We found that $4(2.8 \%)$ were those individuals who were making $50000+$ RS in a month. Significant association of socio economic status with oral cancer has been observed $(\mathrm{P}<0.001) .108(74.48 \%)$ of the participants were illiterate or less than primary pass. Only $3(2.1 \%)$ patients presented with education above grade 16 . Educational Level is significantly associated with Oral Cancer $(P<0.001)$ (table 1).

Table: 1: Socio-demographic characteristics of the study population

\begin{tabular}{|c|c|c|c|}
\hline Characteristics & Variables & $\mathrm{N}=145$ & $(\%)$ \\
\hline \multirow{3}{*}{ Gender } & Male & 92 & 63.4 \\
\hline & Female & 53 & 36.6 \\
\hline & Total & 145 & 100 \\
\hline \multirow{6}{*}{ Age } & 18- 30 years & 13 & 9.0 \\
\hline & $31-40$ years & 33 & 22.8 \\
\hline & 41- 50 years & 43 & 29.7 \\
\hline & $51-60$ years & 38 & 26.2 \\
\hline & 60 and Above & 18 & 12.4 \\
\hline & Total & 145 & 100.0 \\
\hline \multirow{8}{*}{ Education } & Illiterate & 63 & 43.4 \\
\hline & Less than Primary & 8 & 5.5 \\
\hline & $\begin{array}{l}\text { Primary but less than } \\
\text { middle }\end{array}$ & 15 & 10.3 \\
\hline & $\begin{array}{l}\text { Middle but less than } \\
\text { Metric }\end{array}$ & 28 & 19.3 \\
\hline & $\begin{array}{l}\text { Metric but less than } \\
\text { degree }\end{array}$ & 27 & 18.6 \\
\hline & Degree $\&$ above & 3 & 2.1 \\
\hline & Madrassa & 1 & .7 \\
\hline & Total & 145 & 100 \\
\hline \multirow{5}{*}{ Income Level } & Un employed & 50 & 34.5 \\
\hline & $<10000$ & 47 & 32.4 \\
\hline & $10000-25000$ & 35 & 24.1 \\
\hline & $25001-50000$ & 9 & 6.2 \\
\hline & $50000+$ & 4 & 2.8 \\
\hline Total & & 145 & 100 \\
\hline
\end{tabular}

On asking the participant whether or not they knew any friend or family member suffering from oral cancer, majority of the patients, $127(87.6 \%)$ patients replied in negative. The earliest symptom noticed by majority, i.e. $60(41.4 \%)$ patients was non healing ulcer or sore.

In the current study 106 (73.1\%) patients did not get selfmedication and sought a general practitioner. whereas $38(26.2 \%)$ patients identified sought treatment from a 
homeopath or a spiritual healer for around a month. After the first sign appeared majority of the participants $101(69.7 \%)$ consulted the General Practitioner in their neighborhood and $18(12.5 \%)$ visited General dentist, 17 $(11.7 \%)$ went to the ENT surgeon only $4(2.8 \%)$ preferred oncologist and $5(3.4 \%)$ approached Homeopath or spiritual healer. 81 (55.9\%).

102 patients $(70.3 \%)$ presented at advanced stage, i.e. stage III and IV. Only 9 (6.2\%) presented at the initial stage $(P=0.001)$ (table 2$)$.

Table 2: Duration and stage of the lesion at the time of presentation

\begin{tabular}{|l|l|l|l|}
\hline \multirow{5}{*}{ Duration } & Variable & Number & Percentage \\
\cline { 2 - 4 } & Within 14 days & 31 & 21.4 \\
\cline { 2 - 4 } & Within 30 Days & 50 & 34.5 \\
\cline { 2 - 4 } & Within 6 Months & 51 & 35.2 \\
\cline { 2 - 4 } & After 6 Months & 13 & 9 \\
\cline { 2 - 4 } & Total & 145 & 100 \\
\hline \multirow{5}{*}{ Stage } & Stage & Frequency & Percentage \% \\
\cline { 2 - 4 } & Stage 0 & 2 & 1.4 \\
\cline { 2 - 4 } & Stage 1 & 7 & 4.8 \\
\cline { 2 - 4 } & Stage II & 34 & 23.4 \\
\cline { 2 - 4 } & Stage III & 22 & 15.2 \\
\cline { 2 - 4 } & Stage IV & 80 & 55.1 \\
\cline { 2 - 4 } & Total & 145 & 100 \\
\hline
\end{tabular}

Total $81(55.9 \%)$ patient approached health care provider within 30 days, 51 (35.2\%) delayed up to 6 month and $13(9 \%)$ waited for more than 6 months. Majority, i.e. 123 patients $(84.8 \%)$ reported that awareness regarding oral cancer can be spread in our population through media.

We found that (139) $95.86 \%$ of the patients were involved in any habit, that may be Pan, Chalia, Supari, Gutka , Naswar , Alcohol, Cigarette or combination such things. Most of the Patients had habit of PAN or Supari. Gutka , and Main Puri account for $88(60.60 \%)$ of the cases. Out of 145 cancer patients $44(30.34 \%)$ had habit of smoking cigarette and $5 \%(8)$ had history of alcohol intake (Table 3)

Table 3: Addiction history of the oral cancer patients.

\begin{tabular}{|l|l|l|l|}
\hline \multirow{5}{*}{} & Variable & N & \% of the Total \\
\cline { 2 - 4 } & Pan/Chalia/ Supari/ & 116 & $80 \%$ \\
\cline { 2 - 4 } Addiction & Gutka/Mawa/ Mainpuri & 88 & 60.68 \\
\cline { 2 - 4 } & Cigarette & 44 & $30.34 \%$ \\
\cline { 2 - 4 } & Naswaar & 13 & $8.96 \%$ \\
\cline { 2 - 4 } & Alcohol & 8 & $5.51 \%$ \\
\cline { 2 - 4 } & Others & 17 & 11.72 \\
\cline { 2 - 4 } & All of the Above & 139 & 95.86 \\
\hline
\end{tabular}

Buccal Mucosa or Cheeks were found to be the most prevalent region for oral cancer that accounts for 77 $(53 \%)$ of the cases and second most common site was Tongue with $28(19.3 \%)$ participants. $13(9 \%)$ had lesion on the lips. Remaining 27 (18.6\%) of the patients had lesions on different location including Palate, Mandible, Maxilla etc. Significant number i.e. 79 (54.5\%) of Oral cancer thought that the lesion is something usual and not dangerous and would get better itself. Another barrier was the cost of treatment and checkup, 10 (6.9\%) delayed just because of this reason. Fear of biopsy, difficulty in getting the appointment and transportation issues were another factors in delaying to seek the advice. Small number of participant were afraid of the outcome of diagnosis and were too busy so they avoid their lesion (figure 1).

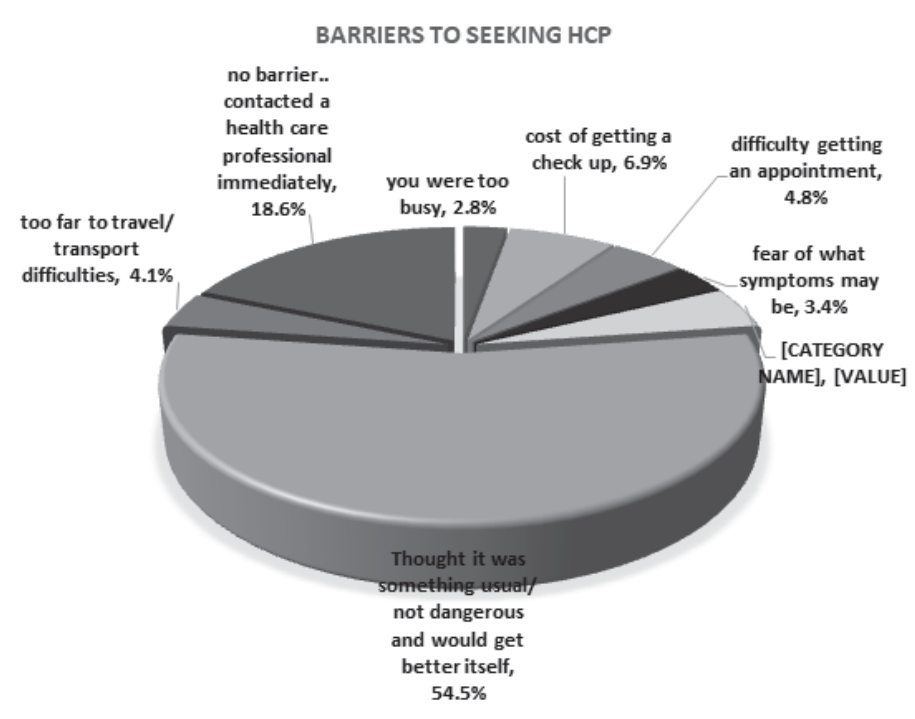

Figure-1: Barriers to seeking HCP

Discussion

Clinical Staging is the most important diagnostic and predictive feature in patients with oral malignant lesions. Although it seems logical that delayed presentation or professional delay by the health care professional leads to diagnosis of advanced stage disease, not all studies supported this finding. Some studies perceived that elements usually linked with patient and professional delays, such as gender, dental status, alcohol consumption, and socioeconomic status, are related to clinical stage at diagnosis9. A study was conducted in Great Britain to determine public awareness and knowledge of oral cancer. They concluded that awareness among public regarding oral cancer was quite less compared to other malignancies, i.e., skin, lung, cervical and this may lead to the late presentation 16 . Majority of the patients who presented with oral cancer in our hospital were male. Due to the differences in habits the occurrence of oral cancer among males is higher 17 . The results are similar to a study conducted at Liverpool and in Karachi had the similar findings 18 19, moreover during the data collection females were also reluctant to be incorporated in the current study due to the cultural norms and personnel issues. Patients with bad financial conditions, less literacy levels delays in the presentation of the disease to the concern person20. This may postulate an association between patient delay and awareness of signs, indications, symptoms and stages of health awareness, however the relationship between low socioeconomic status and delay may also be due to hindrance in getting leave and deduction of the salary from work for those working on daily wages or laboring in manual occupations21. Higher number of participant consulted general physicians, previous 
studies reported the same scenario that patients prefer their family doctor $18,19$.

In our study the most common presenting tumor site was cheek, same findings were reported in another study conducted in Pakistan22. Some patients were aware that oral cancer existed, as they knew of friends or family member suffering from oral malignancy. Despite this, some patients reported that they had no knowledge about the disease. According to them, the best way to get patients to present earlier was through improved awareness of the disease, as many felt there was a gross lack of knowledge in this field of cancers compared to other cancers. On noticing the symptom, majority of the patients sought a general practitioner and not the specialist as they didn't have any knowledge regarding the severity of the disease. As early cancer symptoms aren't unique in appearance, and are usually asymptomatic, it is difficult to distinguish them from usual oral complaints. Tumor symptomology may play a significant role in the diagnostic process, given its effect on attention by the patient and professional care 9 .

A few interviewees consulted a homeopath and took treatment from them for a short period of time. According to the patients, they would have sought advice from a specialist rather than seeking a general practitioner, homeopath or a chemist if they had been aware about the signs and symptoms and severity of the disease. Despite of the these findings, it is crucial that due to their great approachability, pharmacists, homeopath and their staff are made aware regarding head and neck cancer and have increased alliance with the oral healthcare team to achieve the objective of earlier referral to the specialist. Multiple studies proved that diagnosis of the disease may result in good prognosis 23 , 24.

\section{Conclusion}

This study concluded that the first symptom noted by majority was a non-healing ulcer or sore that patients thought was nothing unusual and would get better by itself. The first health care professional contacted was a general practitioner. Major delay was due to patients in seeking a specialist rather than professional delay.

\section{References}

1. Parkin DM, Bray F, Ferlay J, Pisani P. Global cancer statistics, 2002. CA: a cancer journal for clinicians. 2005;55(2):74-108.

2. Vigneswaran N, Williams MD. Epidemiologic trends in head and neck cancer and aids in diagnosis. Oral and Maxillofacial Surgery Clinics. 2014;26(2):123-41.

3. Warnakulasuriya S. Global epidemiology of oral and oropharyngeal cancer. Oral oncology. 2009;45(4):309-16.

4. Bhurgri Y, Bhurgri A, Usman A, Pervez S, Kayani $\mathrm{N}$, Bashir I, et al. Epidemiological review of head and neck cancers in Karachi. Asian Pacific Journal of Cancer Prevention. 2006;7(2):195.

5. Bhurgri Y, Bhurgri A, Hassan SH, Zaidi S, Rahim
A, Sankaranarayanan R, et al. Cancer incidence in Karachi, Pakistan: first results from Karachi cancer registry. International journal of cancer. 2000;85(3):325-9.

6. Bhurgri Y, Rahim A, Bhutto K, Bhurgri A, Pinjani $P$, Usman A, et al. Incidence of carcinoma of the oral cavity in Karachi-District South. JOURNALPAKISTAN MEDICAL ASSOCIATION. 1998;48:321-4.

7. Rogers SN, Brown JS, Woolgar JA, Lowe D, Magennis P, Shaw RJ, et al. Survival following primary surgery for oral cancer. Oral oncology. 2009;45(3):201-11.

8. Khandekar S, Bagdey P, Tiwari R. Oral cancer and some epidemiological factors: a hospital based study. Indian J Community Med. 2006;31(3):157-9.

9. Aquilina PJ. An Investigation into Patient \& Professional Delays in the Diagnosis of Head \& Neck Cancer.

10. Kumar S, Heller R, Pandey U, Tewari V, Bala N, Oanh K. Delay in presentation of oral cancer: a multifactor analytical study. National Medical Journal of India. 2001;14(1):13-7.

11. Yeole B B, R a m a n a k m a r A V, Sankaranarayanan R. Survival from oral cancer in Mumbai (Bombay), India. Cancer Causes \& Control. 2003;14(10):945-52.

12. Güneri $P$, Çankaya $H$, Yavuzer $A$, Güneri $E A$, Eri?en L, Özkul D, et al. Primary oral cancer in a Turkish population sample: association with sociodemographic features, smoking, alcohol, diet and dentition. Oral oncology. 2005;41(10):1005-12.

13. Munck K, Ali MJ, Murr AH, Goldberg AN. Impact of socioeconomic status on the diagnosis to treatment interval in Waldeyer's ring carcinoma. The Laryngoscope. 2005;115(7):1283-7.

14. Davis GE, Bryson CL, Yueh B, McDonell MB, Micek MA, Fihn SD. Treatment delay associated with alternative medicine use among veterans with head and neck cancer. Head \& neck. 2006;28(10):926-31 .

15. Edge SB, Compton CC. The American Joint Committee on Cancer: the 7th edition of the AJCC cancer staging manual and the future of TNM. Annals of surgical oncology. 2010;17(6):1471-4.

16. Warnakulasuriya $K$, Harris $C$, Scarrott $D$, Watt R, Gelbier S, Peters T, et al. Oral cancer: an alarming lack of public awareness towards oral cancer. British dental journal. 1999;187(6):319.

17. Johnson N, Warnakulasuriya K. Epidemiology and aetiology of oral cancer in the United Kingdom. Community dental health. 1993;10:13.

18. Rogers SN, Vedpathak SV, Lowe D. Reasons for delayed presentation in oral and 
oropharyngeal cancer: the patients perspective. British Journal of Oral and Maxillofacial Surgery. 2011;49(5):349-53.

19. Zahid T, Hussain SI, Siddiqui AH, Junaid ST, Ahmed Z, Noorani MM. Health seeking behavior of oral cancer patients of low socioeconomic status: a cross sectional study in a tertiary care hospital of Karachi. Journal of Dow University of Health Sciences. 2014;8(2).

20. Guo Y, Logan HL, Marks JG, Shenkman EA. The relationships among individual and regional smoking, socioeconomic status, and oral and pharyngeal cancer survival: a mediation analysis. Cancer medicine. 2015;4(10):1612-9.

21. Jones L, Bates G, McCoy E, Bellis MA. Relationship between alcohol-attributable disease and socioeconomic status, and the role of alcohol consumption in this relationship: a systematic review and meta-analysis. BMC Public Health. 2015;15(1):400.

22. Kadri S, Uddin S, Ahmed N, Mahmood T. Malignant head and neck tumours in radiology department JPMC Karachi-a tertiary care experience. J Pak Med Assoc. 2015;65(8):86224.

23. McMichael A. Oral cancer in the Third World: time for preventive intervention? International journal of epidemiology. 1984;13(4):403-5.

24. Boyle P, Macfarlane G, Scully C. Oral cancer: necessity for prevention strategies. The Lancet. 1993;342(8880):1129. 